\title{
Novelty Enhancements in Memory Are Dependent on Lateral Prefrontal Cortex
}

\author{
Mark M. Kishiyama, ${ }^{1}$ Andrew P. Yonelinas, ${ }^{3}$ and Robert T. Knight ${ }^{1,2}$ \\ ${ }^{1}$ Helen Wills Neuroscience Institute and ${ }^{2}$ Department of Psychology, University of California, Berkeley, Berkeley, California 94720, and ${ }^{3}$ Department of \\ Psychology, University of California, Davis, Davis, California 95616
}

\begin{abstract}
Physiological evidence indicates that several brain regions, including the medial temporal lobes and prefrontal cortex (PFC), are involved in processing events that are novel or distinctive in their immediate context. However, behavioral studies that investigate whether these regions are critical for producing stimulus novelty advantages in memory are limited. For example, evidence from an animal lesion study indicated that the PFC is involved in stimulus novelty effects, but this has not been examined in humans. In the current study, we used a von Restorff novelty paradigm to test a large cohort of lateral PFC patients $(n=16)$. We found that patients with lateral PFC damage were impaired in recollection- and familiarity-based recognition, and they did not exhibit a normal memory advantage for novel compared with non-novel items. These results provide neuropsychological evidence supporting a key role for the lateral PFC in producing stimulus novelty advantages in memory.
\end{abstract}

\section{Introduction}

The ability to detect novelty is an essential feature of all mammalian nervous systems (Sokolov, 1963), and it plays a critical role in memory in the sense that items that are novel, or distinctive, are remembered better than those that are less distinct (von Restorff, 1933). Although there are various ways in which an item may be novel, here we focus on "stimulus novelty" that refers to cases in which a stimulus is distinctive or unusual relative to other items in the immediate or local context (Kishiyama and Yonelinas, 2006).

Two of the primary methods for examining the effects of stimulus novelty are the novelty oddball (Courchesne et al., 1975) and von Restorff paradigms (von Restorff, 1933). In a novelty oddball paradigm, subjects are presented with infrequent target and lowprobability novel events embedded in a series of repetitive background stimuli. Evidence from a number of novelty oddball studies indicates that novel events activate a distributed novelty detection network. For example, event-related potential (ERP) findings from patients with focal lesions or implanted electrodes suggest that regions in medial temporal lobes (MTL) and prefrontal cortex (PFC) constitute critical components of this network (Knight, 1984, 1996; Halgren et al., 1998; Knight and Scabini, 1998; Daffner et al., 2000). In addition, some ERP evidence suggests that the right lateral PFC and particularly the dorsolateral PFC (DLPFC) may be important for novelty processing (Alexander et al., 1995; Knight and Scabini, 1998; Daffner et al., 2000). However, because memory was not typically measured in

Received Nov. 14, 2008; revised April 15, 2009; accepted May 22, 2009.

This work was supported by National Institute of Neurological Disorders and Stroke Grants NS21135 and PO 40813 and National Institute of Mental Health Grant MH059352. We thank Donatella Scabini for all her help with patient delineation and recruitment and Clay Clayworth for his help with the lesion reconstructions. We are also grateful to all patients and control subjects for their enthusiastic participation.

Correspondence should be addressed to Mark M. Kishiyama, Helen Wills Neuroscience Institute, 132 Barker Hall, MC\#3190, Berkeley, CA 94720-3190. E-mail: mmkishiyama@berkeley.edu.

D01:10.1523/JNEUROSCI.5507-08.2009

Copyright $\odot 2009$ Society for Neuroscience $\quad$ 0270-6474/09/298114-05\$15.00/0 these studies, they do not reveal whether MTL and PFC regions are critical for producing stimulus novelty effects in memory.

In the von Restorff paradigm, subsequent memory for novel and non-novel items is directly contrasted. In this paradigm, subjects study a list of items that includes a subset of items that are made novel or distinctive from the majority. In subsequent tests of memory, subjects are found to exhibit enhanced memory for the novel items. This effect is extremely robust, and it is one of the most widely used methods for examining the effects of stimulus novelty on subsequent memory (for review, see Wallace, 1965; Schmidt, 1991). In a von Restorff study of amnesic patients, Kishiyama et al. (2004) found that stimulus novelty effects were eliminated in patients with MTL lesions, including patients thought to have relatively selective hippocampal damage. Evidence from an animal lesion study revealed that von Restorff or stimulus novelty effects were eliminated in a group of nonhuman primates with lesions to the lateral PFC (Parker et al., 1998). However, the role of the lateral PFC in producing stimulus novelty effects in humans has not been examined.

We used a von Restorff paradigm and a large cohort of lateral PFC patients to examine the role of PFC in producing stimulus novelty advantages in memory. Evidence from previous studies indicates that stimulus novelty benefits both recollection- and familiarity-based recognition in neurologically healthy subjects (Kishiyama and Yonelinas, 2003). We used the same test procedure in the current study to determine whether lateral PFC patients would have reduced stimulus novelty effects in measures of recollection and familiarity compared with age-matched control subjects. In addition, we contrasted patients with different PFC lesions in an effort to examine the contributions of specific PFC subregions in producing stimulus novelty effects in memory.

\section{Materials and Methods}

Subjects. A total of 16 unilateral PFC (9 left, 7 right) patients ( 6 females, 10 males; mean age $=63 \pm 12.2$ years; mean education $=14.5 \pm 2.9$ 
Table 1. Lesion volume and Brodmann's area locations for individual patients

\begin{tabular}{lcl}
\hline Patient & Lesion volume $\left(\right.$ in $\left.\mathrm{cm}^{3}\right)$ & Brodmann's areas \\
\hline 1 & 22.8 & $44,45,46$ \\
2 & 250.5 & $6,8,9,44,45,46$ \\
3 & 34.8 & $8,9,44,45,46$ \\
4 & 338.4 & $6,8,9,10,44,45,46,47$ \\
5 & 43.5 & $9,44,45,46$ \\
6 & 296.7 & $6,8,9,44,45,46$ \\
7 & 77.7 & $6,8,9,44,45,46$ \\
8 & 25.5 & $9,10,46$ \\
9 & 418.5 & $6,8,9,44,45,46$ \\
10 & 24 & $44,45,46$ \\
11 & 142.2 & $6,8,9,10,44,45,46,47$ \\
12 & 9 & $8,9,46$ \\
13 & 45.9 & $8,9,46$ \\
14 & 109.8 & $6,8,46$ \\
15 & 38.7 & $6,8,9,46$ \\
16 & 143.4 & $8,9,10,44,45,46$ \\
\hline
\end{tabular}

years) and 16 age- and education-matched control subjects ( 9 females, 7 males; mean age $=62.9 \pm 12.4$ years; mean education $=14.6 \pm 2.0$ years) participated. Patients and control subjects did not differ in age $\left(t_{(15)}=0.24, p=0.82\right)$ or education $\left(t_{(15)}=0.11, p=0.91\right)$. Patients were recruited from the Veteran's Administration Northern California Health Care System in Martinez, CA and other participating hospitals and clinics. Patients were included if they were at least 6 months postcerebral vascular accident and had no history of any other medical, neurological or psychiatric disorder. All of the patients had the same etiology of infarction of the precentral branch of the middle cerebral artery. The average lesion volume was $126.3 \mathrm{~cm}^{3}$. The lesions were centered in the lateral PFC encompassing both DLPFC [Brodmann's areas (BA) 9 and 46] and ventrolateral PFC (VLPFC) (BA 44, 45, and 47) subregions with varying degrees of damage in BA 6,8 , and 10 . Note that every patient had damage to at least one DLPFC region (BA 9 and 46) and only five patients (patients 8, 12-15) had damage that did not include at least one VLPFC region (BA 44, 45, and 47). Lesion volume and specific BA locations for individual patients are shown in Table 1. Individual lesion reconstructions and group lesion overlaps are shown in Figure $1 A$ for the left PFC group (patients 1-9) and in Figure $1 B$ for the right PFC group (patients 10-16). The control subjects were recruited from the Davis, Sacramento, and San Francisco Bay Area communities, and they had no history of neurological or psychiatric disorders. Subjects were paid for participation and signed consent statements approved by the Institutional Review Boards of the University of California, Davis, the University of California, Berkeley, and the Veterans Administration Research Service.

Procedure. A total of 660 thumbnail object images from MasterClips Premium Image Collection (MasterClips, 1998) were used as study and test items. The objects were presented in red against a white background or in yellow against a black background. Subjects were told that a long series of objects would be presented on the computer monitor at a rapid rate, and that they were to try to remember them for a later memory test. They were told that some of the objects would appear in red and some would appear in yellow, but that color was not important and that they were to try to remember all the objects. During the study phase, subjects were presented with 600 items at a rate of $850 \mathrm{~ms}$ per item. Within the study list, there were 60 critical study items; 30 were novel and 30 were non-novel items appearing in red and yellow. There were 540 filler items appearing in the same color as the non-novel study items. Items were thus made novel in the sense that they appeared in a less frequent color than the majority of items in the study list (Fig. $2 A$ ). Items were presented in a pseudo-random order such that novel objects were separated by a minimum of 10 intervening non-novel objects. Novel and nonnovel items were counterbalanced across subjects.

At test, subjects were presented with 120 items one at a time, 30 "old" novel items presented in the original color, as well as 30 "new" items presented in the same color as the novel items, mixed with 30 old nonnovel items presented in their original color, as well as 30 new items presented in the same color as the non-novel items. For each item, subjects were required to make a "remember," "know," or "new" judgment, and the experimenter recorded the responses. The remember-know instructions were adapted from previous studies (Tulving, 1985; Gardiner, 1988). Subjects were told that they were to respond remember if they could recollect some qualitative information about the study event. Moreover, they were instructed that they should only respond remember if they could, if asked, tell the experimenter what they recollected about that study event. Subjects were told to respond know if they thought the item was studied, but they could not recollect any details about the study event. That is, they should respond know if the item was familiar but not recollected. They were told to respond new if they thought the item was not in the study list. To ensure that subjects understood the test instructions, they were asked to describe the remember-know distinction back to the experimenter, and the instructions were repeated if the subject appeared to misunderstand the distinction.

Because subjects were instructed to respond remember whenever an item was recollected, the probability of a remember response was used as an estimate of recollection (i.e., $R=$ remember). To incorporate false alarm rates, accuracy was measured using $d$, such that the proportion of correct and incorrect remember responses were used as hits and false alarms. Because subjects were instructed to respond know when an item was familiar in the absence of recollection [i.e., know $=F(1-R)$ ], familiarity was estimated as the probability of a know response given the item was not recollected [i.e., $F=$ know/ $(1-R)$ ]. As with the recollection estimates, the estimates of familiarity for old and new items were used as hits and false alarms to derive $d^{\prime}$ estimates of familiarity accuracy.

\section{Results}

Table 2 presents the mean proportion of remember and know responses for the left and right PFC patients and the control subjects. Estimates of recollection and familiarity were obtained from remember-know responses (Yonelinas and Jacoby, 1995). The estimates were reported in $d^{\prime}$ values (MacMillan and Creelman, 1991) - derived from the proportions of hit and false alarm rates for remember and know responses (see Materials and Methods for details). Estimates of recollection and familiarity for novel and non-novel items for left and right lateral PFC patients and control subjects are shown in Figure 2B. All measurements were analyzed using Greenhouse-Geisser corrected, repeatedmeasures ANOVA. Planned $t$ tests were one-tailed.

Recognition memory performance was significantly lower in the PFC patients compared with the control subjects $\left(F_{(1,30)}=\right.$ $5.87, p=0.02$ ), and planned contrasts revealed that these deficits were apparent in both recollection $\left(t_{(15)}=1.74, p=0.05\right)$ and familiarity estimates $\left(t_{(15)}=1.84, p=0.04\right)$. In addition, planned contrasts revealed that there was a significant stimulus novelty advantage in recollection for the control subjects $\left(t_{(15)}=1.90\right.$, $p=0.04)$, whereas there was no stimulus novelty advantage in recollection for the lateral PFC patients $\left(t_{(15)}=0.55, p=0.29\right)$. In fact, the patients' recollection estimates were slightly higher for the non-novel compared with the novel items (Fig. 2B). Note, however, that the group $\times$ stimulus interaction did not quite reach the level of statistical significance $\left(F_{(1,30)}=3.19, p=0.08\right)$. Estimates of familiarity were generally greater for the novel compared with the non-novel items, but these differences were not significant for either the control subjects or the PFC patients (all $p>0.05)$.

Additional comparisons revealed that there were no significant recognition differences between the patients with left (patients 1-9) and right lateral PFC lesions (patients 10-16). In general, the patients with right PFC lesions performed slightly more poorly than the left PFC patients, possibly because of the visual nature of the stimuli (Milner et al., 1991), but these differences were not significant in recollection $\left(t_{(11)}=0.83, p=0.21\right)$ or 
A

\section{Left Frontals}

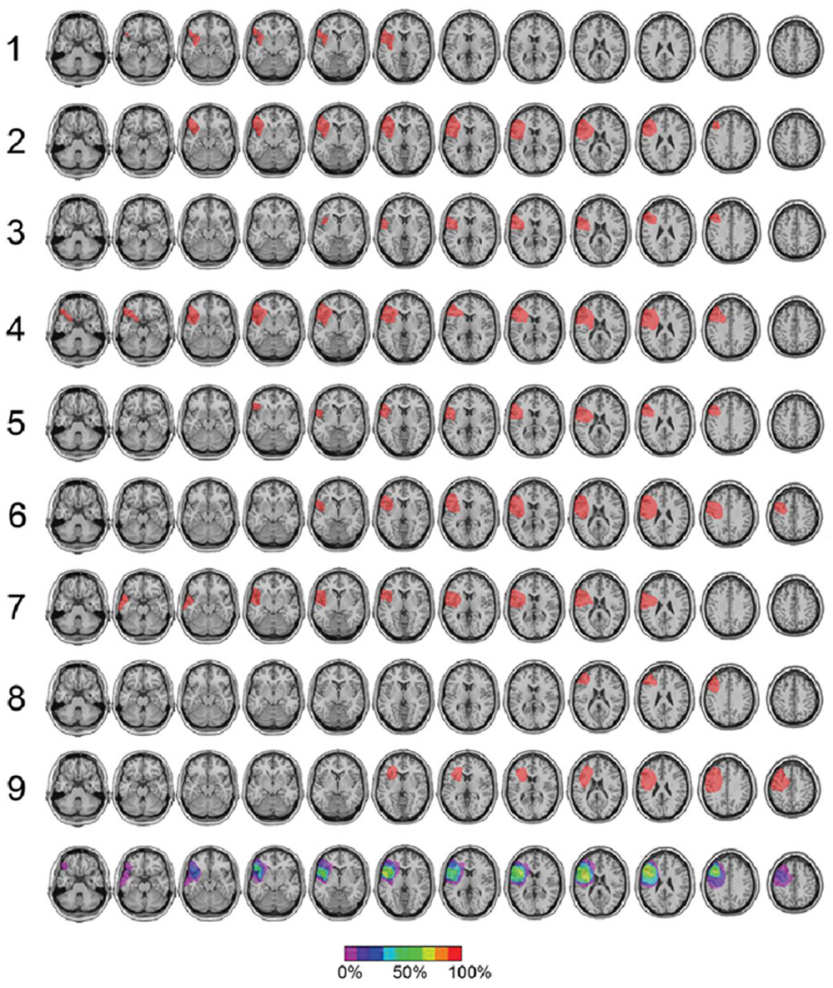

B

Right Frontals

Figure 1. A, Lesion reconstructions for individual patients with left frontal lesions (patients 1-9). A group lesion overlap is shown at the bottom of the figure. The scale indicates the percentage of patients with lesions in a specific area. $\boldsymbol{B}$, Lesion reconstructions for individual patients with right frontal lesions (patients 10-16). A group lesion overlap is shown at the bottom of the figure. The scale indicates the percentage of patients with lesions in a specific area.

familiarity $\left(t_{(12)}=1.26, p=0.12\right)$. Moreover, there were no stimulus novelty effects on recollection in either the left $t_{(8)}=$ $0.58, p=0.29)$ or the right PFC patients $\left(t_{(6)}=0.12, p=0.45\right)$. As with the control subjects, there were numerical stimulus novelty advantages in the familiarity estimates for both the left and right PFC groups (Fig. 2B). This difference was not significant in the right PFC group $\left(t_{(6)}=0.59, p=0.29\right)$, but approached significance in the left PFC group $\left(t_{(8)}=1.84, p=0.05\right)$.

A final comparison was made between patients with damage limited to the DLPFC region (the DLPFC group; patients 8, 1215) and patients with damage that included the DLPFC and VLPFC regions (the DLPFC + VLPFC group; all remaining patients). The analysis indicated that recognition estimates did not differ between these groups for recollection $\left(t_{(7)}=0.14, p=0.45\right.$ ) or familiarity $\left(t_{(12)}=1.03, p=0.16\right)$. In addition, stimulus novelty effects on recollection were not seen in the DLPFC patients $\left(t_{(4)}=0.57, p=0.30\right)$ or the DLPFC + VLPFC patients $\left(t_{(10)}=\right.$ $0.29, p=0.39)$. There were stimulus novelty advantages in familiarity in the DLPFC + VLPFC patients $\left(t_{(10)}=2.73, p=0.01\right)$, but these advantages were absent in the DLPFC patients $\left(t_{(4)}=\right.$ $0.03, p=0.49)$. However, the former finding could reflect the larger sample size of this group.

\section{Discussion}

The PFC has been implicated in a number of studies of stimulus novelty. For example, evidence from studies using ERPs, depth electrodes in humans, and single-unit studies in monkeys suggests that the PFC plays an important role in novelty detection (Knight, 1984; Halgren et al., 1998; Daffner et al., 2000; Matsu- moto et al., 2007). In addition, evidence from lesion, ERP, and functional magnetic resonance imaging studies indicate that both the PFC and MTL are thought to constitute critical components of a distributed novelty detection network (Knight, 1996, 1997; McCarthy et al., 1997; Knight and Scabini, 1998; Strange and Dolan, 2001; Yamaguchi et al., 2004). However, these studies do not reveal whether these regions are critical for producing stimulus novelty effects in memory, because memory was not typically measured in these studies.

In the current study, we used a von Restorff paradigm to investigate the critical role of the lateral PFC in stimulus novelty and memory. In particular, if the lateral PFC is essential for novelty-related memory enhancements, then lesions in this area should lead to reductions in stimulus novelty effects in memory. We found that novelty effects on recollection that were observed in control subjects were absent in lateral PFC patients. Similar results have been observed in nonhuman primates (Parker et al., 1998). The current findings suggest that the human lateral PFC may be important for producing stimulus novelty effects in memory. Together with the results from a study of patients with MTL damage (Kishiyama et al., 2004), these findings provide support for anatomically motivated memory models of novelty suggesting that novelty advantages in memory reflect interactions between memory mechanisms in the MTL and novelty monitoring mechanisms in the PFC (Metcalfe, 1994; Tulving et al., 1994; Tulving and Kroll, 1995). Moreover, the current findings extend these models by implicating the lateral PFC and the hippocampus. 


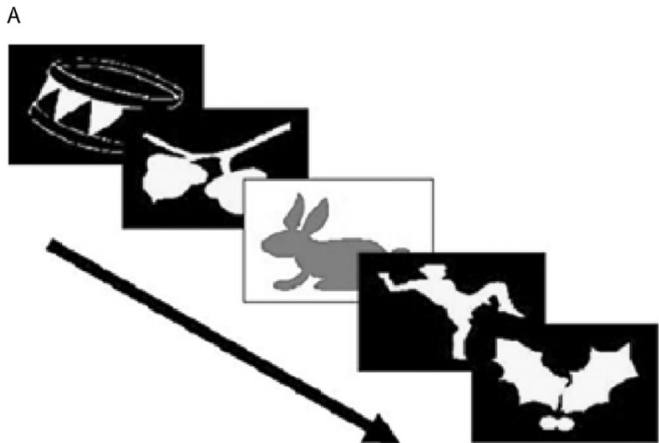

B

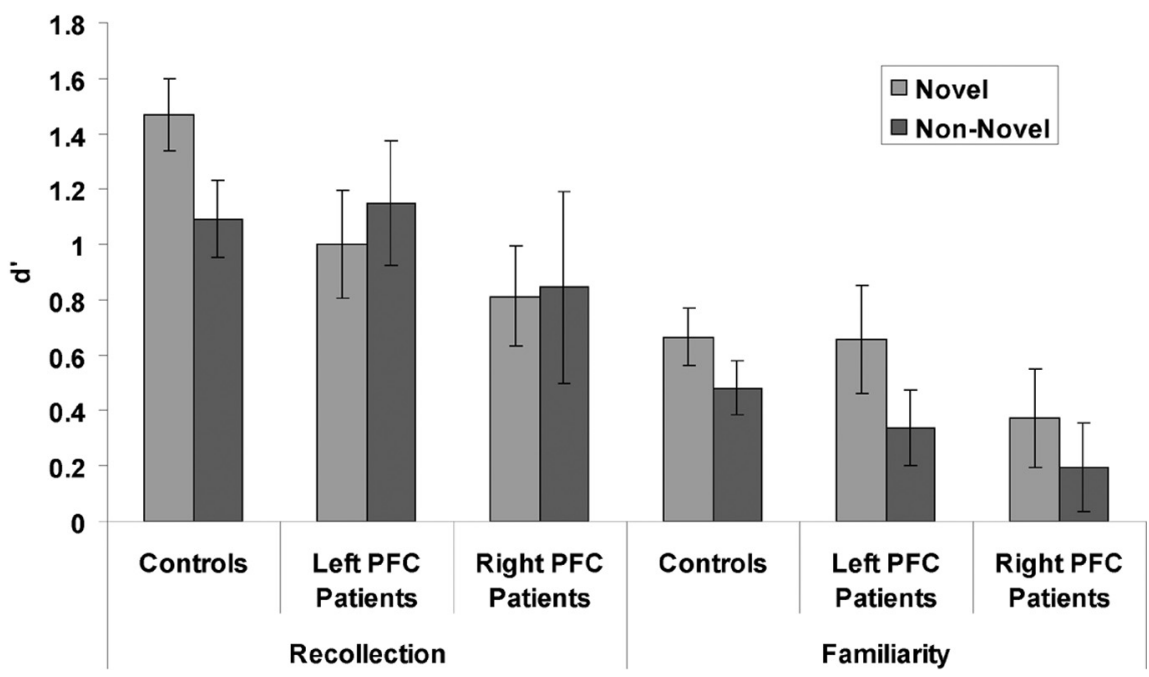

Figure 2. $\quad \boldsymbol{A}$, The von Restorff paradigm. The novel item appears in red (shown here in gray) against a white background and the non-novel items appear in yellow (shown here in white) against a black background. $\boldsymbol{B}$, Recollection and familiarity estimates for novel and non-novel items for left and right lateral PFC patients and control subjects.

Table 2. The probabilities of remember $(R)$ and know $(K)$ responses

\begin{tabular}{|c|c|c|c|c|}
\hline & \multicolumn{2}{|l|}{ Old items } & \multicolumn{2}{|l|}{ New items } \\
\hline & $\mathrm{R}$ & K & $\mathrm{R}$ & K \\
\hline \multicolumn{5}{|c|}{ Controls $(n=16)$} \\
\hline Novel & $0.49(0.16)$ & $0.20(0.14)$ & $0.07(0.05)$ & $0.18(0.15)$ \\
\hline Non-novel & $0.50(0.19)$ & $0.26(0.16)$ & $0.18(0.18)$ & $0.28(0.15)$ \\
\hline \multicolumn{5}{|c|}{ Left PFC patients $(n=9)$} \\
\hline Novel & $0.29(0.16)$ & $0.27(0.20)$ & $0.04(0.05)$ & $0.15(0.08)$ \\
\hline Non-novel & $0.36(0.20)$ & $0.24(0.15)$ & $0.06(0.06)$ & $0.23(0.11)$ \\
\hline \multicolumn{5}{|c|}{ Right PFC patients $(n=7)$} \\
\hline Novel & $0.30(0.14)$ & $0.13(0.11)$ & $0.11(0.13)$ & $0.10(0.09)$ \\
\hline Non-novel & $0.40(0.26)$ & $0.18(0.14)$ & $0.17(0.20)$ & $0.19(0.09)$ \\
\hline
\end{tabular}

SDs are presented in parentheses.

The current findings also provide insights into the regions within the PFC that may be important for producing stimulus novelty advantages in memory. Although evidence from ERP studies suggested that the right lateral PFC and the DLPFC may be particularly important for novelty processing (Alexander et al., 1995; Knight and Scabini, 1998; Daffner et al., 2000), the current findings suggest that stimulus novelty advantages observed in recognition memory are not strongly lateralized to the left or right hemisphere. In addition, the current results suggest that damage to the DLPFC is sufficient to eliminate the novelty advantages in memory.

Although the current results provide important information about the role of the lateral PFC in producing stimulus novelty effects in memory, they are not particularly informative about specific cognitive processes that may support these effects. One possibility is that novel items attract more attention than non-novel items, thus enhancing the encoding of these items. Another possibility is that stimulus novelty may benefit retrieval by either enhancing monitoring processes or reducing interference for these items at time of test (Kishiyama and Yonelinas, 2006). Future investigations using neuroimaging techniques could be helpful in determining the contribution of these processes.

There is some evidence indicating that acetylcholine (ACh) may be the neurochemical mechanism that supports interactions across a distributed prefrontalhippocampal network that may underlie both novelty detection and stimulus novelty effects in memory. That is, ACh plays a major role in both memory (Hasselmo, 2006) and novelty detection (Yamaguchi et al., 2004). Basal forebrain cholinergic neurons project to cortical regions that include the lateral PFC and the hippocampus. Disconnection of prefrontal and temporal cortical regions from ACh afferents has been shown to lead to deficits in visual recognition memory (Easton et al., 2001). In addition, evidence from animal studies reveals that cholinergic neurons projecting to PFC and the hippocampus are activated by novel stimuli (Acquas et al., 1996). Moreover, administration of the ACh antagonist scopolamine has been found to reduce both memory performance and the frontal P3 response (Potter et al., 2000). Cortical modulation of ACh in the basal forebrain could therefore underlie modulation of both attentional resource allocation involved in novelty detection and the encoding of novel events (Ranganath and Rainer, 2003).

The current results also revealed that overall recognition memory performance was reduced in lateral PFC patients compared with control subjects. These findings support previous evidence indicating that PFC plays a critical role in long-term memory (Shimamura, 1995). Planned contrasts revealed that lateral PFC patients had reduced estimates of recollection and familiarity compared with control subjects. These findings are consistent with a previous remember/know study that found recollection and familiarity deficits in lateral PFC patients (Duarte et al., 2005).

In conclusion, the current results demonstrate that the human lateral PFC is important for producing stimulus novelty effects in memory. Combined with previous research, these findings further delineate critical subregions of a distributed novelty processing network, including the hippocampus and the lateral PFC, that support both novelty detection and stimulus novelty advantages in memory.

\section{References}

Acquas E, Wilson C, Fibiger HC (1996) Conditioned and unconditioned stimuli increase frontal cortical and hippocampal acetylcholine release: effects of novelty, habituation, and fear. J Neurosci 16:3089-3096. 
Alexander JE, Porjesz B, Bauer LO, Kuperman S, Morzorati S, O’Connor SJ, Rohrbaugh J, Begleiter H, Polich J (1995) P300 hemispheric asymmetries from a visual oddball task. Psychophysiology 32:467-475.

Courchesne E, Hillyard SA, Galambos R (1975) Stimulus novelty, task relevance and the visual evoked potential in man. Electroencephalogr Clin Neurophysiol 39:131-143.

Daffner KR, Mesulam MM, Scinto LF, Acar D, Calvo V, Faust R, Chabrerie A, Kennedy B, Holcomb P (2000) The central role of the prefrontal cortex in directing attention to novel events. Brain 123:927-939.

Duarte A, Ranganath C, Knight RT (2005) Effects of unilateral prefrontal lesions on familiarity, recollection, and source memory. J Neurosci 25:8333-8337.

Easton A, Parker A, Gaffan D (2001) Crossed unilateral lesions of medial forebrain bundle and either inferior temporal or frontal cortex impair object recognition memory in Rhesus monkeys. Behav Brain Res 121:1-10.

Gardiner JM (1988) Functional aspects of recollective experience. Mem Cognit 16:309-313.

Halgren E, Marinkovic K, Chauvel P (1998) Generators of the late cognitive potentials in auditory and visual oddball tasks. Electroencephalogr Clin Neurophysiol 106:156-164.

Hasselmo ME (2006) The role of acetylcholine in learning and memory. Curr Opin Neurobiol 16:710-715.

Kishiyama MM, Yonelinas AP (2003) Novelty effects on recollection and familiarity in recognition memory. Mem Cognit 31:1045-1051.

Kishiyama MM, Yonelinas AP (2006) Stimulus novelty effects on recognition memory: Behavioral properties and neuroanatomical substrates. In: Distinctiveness and memory (Hunt RR, Worthen J, eds), pp 381-404. New York: Oxford UP.

Kishiyama MM, Yonelinas AP, Lazzara MM (2004) The von Restorff effect in amnesia: the contribution of the hippocampal system to noveltyrelated memory enhancements. J Cogn Neurosci 16:15-23.

Knight RT (1984) Decreased responses to novel stimuli after prefrontal lesions in man. Electroencephalogr Clin Neurophysiol 59:9-20.

Knight RT (1996) Contribution of the human hippocampal region to novelty detection. Nature 383:256-259.

Knight RT (1997) Distributed cortical network for visual attention. J Cogn Neurosci 9:75-91.

Knight RT, Scabini D (1998) Anatomic bases of event-related potentials and their relationship to novelty detection in humans. J Clin Neurophysiol 15:3-13.

MacMillan NA, Creelman CD (1991) Detection theory: a user's guide. New York: Cambridge UP.
MasterClips (1998) [Computer software.] San Rafael, CA: IMSI.

Matsumoto M, Matsumoto K, Tanaka K (2007) Effects of novelty on activity of lateral and medial prefrontal neurons. Neurosci Res 57:268-276.

McCarthy G, Luby M, Gore J, Goldman-Rakic P (1997) Infrequent events transiently activate human prefrontal and parietal cortex as measured by functional MRI. J Neurophysiol 77:1630-1634.

Metcalfe J (1994) A computational modeling approach to novelty monitoring, metacognition, and frontal lobe dysfunction. In: Metacognition: knowing about knowing (Metcalfe J, Shimamura AP, eds), pp 137-156. Cambridge, MA: MIT.

Milner B, Corsi P, Leonard G (1991) Frontal-lobe contribution to recency judgments. Neuropsychologia 29:601-618.

Parker A, Wilding E, Ackerman C (1998) The von Restorff effect in visual object recognition memory in humans and monkeys: the role of frontal/ perirhinal interaction. J Cog Neurosci 10:691-703.

Potter DD, Pickles CD, Roberts RC, Rugg MD (2000) Scopolamine impairs memory performance and reduces frontal but not parietal visual P3 amplitude. Biol Psychol 52:37-52.

Ranganath C, Rainer G (2003) Neural mechanisms for detecting and remembering novel events. Nat Rev Neurosci 4:193-202.

Schmidt SR (1991) Can we have a distinctive theory of memory? Mem Cog nit 19:523-542.

Shimamura AP (1995) Memory and frontal lobe function. The cognitive neurosciences (Gazzaniga MS, ed), pp 803-813. Cambridge, MA: MIT.

Sokolov EN (1963) Higher nervous functions: The orienting reflex. Annu Rev Physiol 25:545-580.

Strange BA, Dolan RJ (2001) Adaptive anterior hippocampal responses to oddball stimuli. Hippocampus 11:690-698.

Tulving E (1985) Memory and consciousness. Can Psychol 26:1-12.

Tulving E, Kroll N (1995) Novelty assessment in the brain and long-term memory encoding. Psychon Bull Rev 2:387-390.

Tulving E, Markowitsch HJ, Kapur S, Habib R, Houle S (1994) Novelty encoding networks in the human brain: Positron emission tomography data. Neuroreport 5:2525-2528.

von Restorff H (1933) Uber die wirkung von bereichsbildungen im spurenfeld. Psychol Forsch 18:299-342.

Wallace WP (1965) Review of the historical, empirical, and theoretical status of the von Restorff phenomenon. Psychol Bull 63:410-424.

Yamaguchi S, Hale LA, D’Esposito M, Knight RT (2004) Rapid prefrontalhippocampal habituation to novel events. J Neurosci 24:5356-5363.

Yonelinas AP, Jacoby LL (1995) The relation between remembering and knowing as bases for recognition: Effects of size congruency. J Mem Lang 34:622-643. 